\title{
Prognostication and Risk factors of Status Epilepticus in patients treated in Neurointensive Care Units
}

Emad Fawzy Abdel Monem Shaheen, ${ }^{1}$ MD.

\section{* Corresponding Author:}

Emad Fawzy Abdel Monem Shaheen emad.shahien25@gmail.com

Received for publication July 26, 2021; Accepted September 15, 2021; Published online September 15, 2021.

Copyright The Author published by Al-Azhar University, Faculty of Medicine, Cairo, Egypt. Users have the right to read, download, copy, distribute, print, search, or link to the full texts of articles under the following conditions: Creative Commons AttributionShare Alike 4.0 International Public License (CC BY-SA 4.0).

doi: 10.21608/aimj.2021.83972.1520.

${ }^{I}$ Neurology Department, Faculty of Medicine, Al-Azhar University.

\begin{abstract}
Background: Status epilepticus (SE) can lead to sequelae or even death. Identifying characteristics associated with poor outcome is crucial in guiding patient treatment, to our information, there is no data illustrating the prevalence and outcome of cases with critical tumor illness with AMS accompanied to non-convulsive seizures (NCS) or NCSE

Aim of the study: The aim of the study was to evaluate risk factors and prognosing in cases with Status epilepticus (SE) treated in neurointensive care units.

Patients and Methods: This was a retrospective cohort investigation, in which 50 cases had SE-episodes were included, we in retrospect analyzed all SE-episodes managed over an interval of 2-yrs (April 2019 to April 2021) in the NICU at the hospital.

Results: the commonest cause was idiopathic (40\%), the commonest trigger factor was poor compliance $(38 \%)$. $88 \%$ of the patients were recurrent SE.

Conclusion: Many conditions have complicated the image in daily practice so real-life data are vital so as to recognize real cases in the ward. No severe seasonality of SE patients has been found. In our study, the commonest cause of SE was idiopathic, the commonest trigger factor was poor compliance and most of the patients were recurrent $\mathrm{SE}$,
\end{abstract}

Keywords: Epilepsy; status epilepticus; prognostic factors; neurointensive care units, outcomes.

Disclosure: The authors have no financial interest to declare in relation to the content of this article. The Article Processing Charge was paid for by the authors.

Authorship: All authors have a substantial contribution to the article.

\section{INTRODUCTION}

Previous studies on the epidemiology of SE found incidence rates ranging from 3.5 to 41 per 100000 per year in North America, 9.9 to 27.2 in Europe, 1.3 to 5.2 in Asia, and 10.8 in Africa. ${ }^{1}$

In 2015, the International League Against Epilepsy (ILAE) proposed a new definition of status epilepticus (SE): 5 minutes of ongoing seizure activity to diagnose convulsive SE (CSE, ie, bilateral tonic-clonic SE) and 10 minutes for focal SE and absence SE, rather than the earlier criterion of 30 minutes. Based on semiology, several types of SE with prominent motor phenomena at any time (including CSE) were distinguished from those without (ie, nonconvulsive SE, NCSE) ${ }^{\mathbf{1}}$. Nonconvulsive status epilepticus (NCSE) is existing in 10 to $30 \%$ of Intensive Care Unit (ICU) cases with altered mental status (AMS) and is accompanied to weak outcome, while non-convulsive seizure is present in $14.5 \%$ of cases with critical illness in ICU, NCS and NCSE may be a reason of coma and changed mentation in $18-45 \%$ of cases presented to the ICU. ${ }^{2}$
Status epilepticus (SE) may be fatal or cause dangerous sequelae. The occurrence of SE, conventionally outlined as seizures durable for more than 30-min, the occurrence in accordance to the most recent operative definitions of 5-min period, previously called "impending SE" 3, isn't known. This quicker period limit assists the clinician regarding to when to initiate anti-convulsive intervention. The 30-mins limit will more dependably detect cases with a significant risk of sequelae and mortality till the seizures are ended. ${ }^{4}$

SE is a disorder and utmost extreme form of epilepsy, that causes irregular and extended seizure (at minimum 5-min). If SE perseveres longer 30-min, it can have severe long-term results. regarding the novel SE sorting scheme, there are 2 operative dimensions of the description: time point 1 (T1) is accompanying with oddly extended seizure, when treatment must be started, whereas time point 2 (T2) is correlated to the time of on-going seizure activity including a risk of lasting results. ${ }^{5}$ 
$\mathrm{SE}$ is one of the commonest neurological emergencies. It is a possibly deadly condition that requires a fast and specific intervention so as to avoid cerebral damages because of initial excito-toxicity. Management is vital as GABA sensitivity reductions and the sensitivity to excito-toxic neuro-transmitters rises quickly, parting only a short period for operative intervention. SE can as well have lifetime results and, particularly in intractable SE, the possibility of being epileptic is elevated. ${ }^{6}$

Identification of Predictors for intractable SE is critical for detecting cases at risk early in the course of the disorder. Therefore, tailoring the intervention growth approaches for such cases can, instead, avoid opposing impacts, and instead, decrease the danger for rising longstanding RSE involving its harmful results. Up to now, a retrospective cohort research has proposed that the semiotics of status can comprise prognostic data, with NCSE and focal motor status found significantly more frequently in RSE in comparison to non-RSE (NRSE), while the primary reason of SE didn't have a significant influence on refractoriness. ${ }^{7}$

Many researches have evaluated outcomes in SE generally. Death within 30-days (short-term death) thereafter SE was defined as from 7 to $39 \%$. Morbidity involving severe focal neurologic shortages, cognitive impairments, and advance of epilepsy was found in 3 to $13 \%$ of patients. But systematic predictive information concentrating on RSE are in general missing. Particularly, rate of postSE symptomatically epilepsy is mostly not known and, up to now, haven't been deeply analyzed. ${ }^{8}$

Inopportunely, the description of refractoriness in SE as yet was subject to much disagreement and misperception. In various investigations, in addition to fail of anti-convulsants, a smallest period that has passed since seizure beginning was employed as the cornerstone of the description. But, in others the necessity was the fall of a number of anti-convulsants nevertheless of period of seizure activity, Rebellious $\mathrm{SE}$ has required a agreement definitions; most nowadays respect it as SE that lasts in spite of management with benzodiazepine (benzo) and one anti-epileptic drugs (AEDs). Others respect refractory SE as failures of benzos and $2 \mathrm{AED}$, as: Lorazepam + phenobarb + phenytoin. The $1^{\text {st }}$ description is frequently stated to as 2 AED failures and the $2^{\text {nd }}$ one is labelled the 3 AED failures. ${ }^{9,10}$

The current work aimed to recognize risk factors and outcomes in SE. We matched in retrospect the prognostic characteristics of SE-episodes with those of NSE in cases managed in a neurological-ICU (NICU) over an interval of 2-yrs.

\section{PATIENTS AND METHODS}

The present study was conducted at Al-Azhar University Hospitals. The study protocol was approved by the local ethical committee and all participants provided informed consent. The present operative description of $\mathrm{SE}$ is $\geq 5 \mathrm{~min}$ of nonstop seizure or 2 or more discrete seizures among which there isn't complete consciousness recovery. This time-frame applies to sudden or tonicclonic SE. For focal seizures with consciousness reduction and nonattendance seizures, the time-frame is $10(-15)$ min. ${ }^{4}$

Refractory SE was definite as SE episodes needing 3 or more AEDs for terminating. ${ }^{9}$ The case with refractory CSE treated by propofol as the 3rd-line agent according to our protocol. SE was sorted as either de novo (no prior seizures) or as happening in cases with preceding seizures.

The clinical diagnosing of SE was, in NCSE patients, established by a neurologist. For index refractory CSE episodes, 8/18 have been approved by EEG throughout the ictal phase. An extra 2 out of 18 cases have postictal EEG recordings giving epileptic activities. For cases with no EEG recordings throughout the ictal or postictal phase, 6 out of 8 have preceding or following EEGs with epileptic activities. For index NCSE episodes, 20 out of 39 have been approved by EEGs throughout the ictal stage. A further 8 out of 39 have EEG recordings just afterward end, revealing focal deceleration or focal functional conflicts reliable with the semiotics. Amongst the residual, 8 have preceding recordings with epileptic activities. ${ }^{11}$

We in retrospect analyzed all SE-episodes managed throughout an interval of 2-yrs (April 2019 to April 2021 ) in the NICU at the ..... Hospital.

To confirm identifications of all potential episodes we performed a computer supported searches of cases records via definite keywords e.g., "seizure clustering", "SE", and "prolonged epileptic seizures". Episodes have been involved if SE commenced thereafter admittance to the NICU, earlier admittance but still ongoing afterward admittance to the NICU, and SE ended near-term previous to admittance to the NICU, if the admittance was causally linked to the SE.

\section{Exclusion criteria}

- Episodes have been excepted if the cases reports were not accessible.

- If loss of consciousness among 2 seizures in 5min was iatrogenic

- If the episodes sorted as SE was in retrospect a paroxysmal event of nonepileptic origin - that is, psychogenic nonepileptic seizure, extended convulsive syncopes, temporary ischemic attacks, etc.

The cases with "de novo" SE have been followedup using phone calls regarding the progress of symptoms of epilepsy afterward SE. Previously the phone call cases have been communicated via mail giving them the option to reject the interviews. 
The local ethics committee of the accepted the procedures and written agreement has been attained from the access taking part in the following-up investigation.

\section{Clinical data}

To analyze the clinical parameters, we used structured data collecting grid that has been employed by 2 non-dependent reviewers.

For all SE-episodes, the case's demographic information (ages, gender) and medical history (acute/chronical, neurologic/non-neurologic) have been recorded. Data on one-on-one semiology, aetiology, and clinical courses have been assessed. Furthermore, para-clinical information from the 1st day afterward the beginning of SE involving serum sodium and glucose level, rectal temp, and CSF parameters have been analyzed.

\section{Outcome Measures}

Lastly, we analyzed the outcomes involving SEperiod, short-term re-occurrence of epileptic activities in 24-h thereafter SE end, hospitalization period and in the NICU, in hospital death, and, in cases with "de novo" SE, progress of symptoms epilepsy.

As the prognosing of complex partial-SE (CPSE) and generalized convulsive-SE (GCSE) might vary, subgroup analyzing of these 2 kinds of SE regarding outcomes was done.

For the NCSE group, the outcomes substitutes were mortality, severe sequelae (long-term and highly impacting everyday living), moderate sequelae (severe, long-lasting for $>1$-mth, but not long-term or long-term with a minor or moderate influence on everyday living), mild sequelae (small and temporary or more serious permanent for semiology, were therefore involved with no EEG approval.

\section{Statistical analysis}

Collected data have been analyzed via SPSS 20.0. Frequency distributions of predictive and prognostic parameters of RSE and NRSE were matched so as to recognize features of RSE and have been counted via the $\mathrm{x} 2$ testing. The $\mathrm{t}$ testing has been utilized for analysis of continuous data with normal distributions and the Mann-Whitney $U$ testing for data with nonnormal distributions. Where applicable, Pearson's association coefficient has been estimated. Results have been considered significant at p-value 0.05 .

\section{RESULTS}

Table 1: Basic features of the cases and it shows that $58 \%$ of the patients were males, $82 \%$ were urban, and $44 \%$ were married. $74 \%$ of the cases have epilepsy history.

Table 2: Clinical data of SE and it shows that the commonest cause was idiopathic (40\%), the commonest trigger factor was poor compliance (38\%). $88 \%$ of the patients were recurrent SE.

Table 3: Comparison between patients with SE regarding epilepsy history, shows

A high significant change was found among the groups regarding causes and Trigger factors

Table 4: Distribution of studied group as regard to epilepsy history, shows that Among patients with epilepsy history, $65 \%$ were generalized, $48.6 \%$ were on mono AED and the commonest etiology was idiopathic (54\%).

Table 5: Individual etiologies of SE

\begin{tabular}{|l|l|c|}
\hline \multirow{2}{*}{ Age } & Mean \pm SD & Total $(\mathrm{n}=50)$ \\
\cline { 2 - 3 } & Range & $36.99 \pm 17.15$ \\
\hline \multirow{3}{*}{ Sex } & Females & $5-72$ \\
\cline { 2 - 3 } & Males & $21(42 \%)$ \\
\hline \multirow{2}{*}{ Mesidence } & Rural & $29(58 \%)$ \\
\cline { 2 - 3 } & Urban & $9(18 \%)$ \\
\hline \multirow{3}{*}{ Sexual history } & No & $41(82 \%)$ \\
\cline { 2 - 3 } & Yes & $28(56 \%)$ \\
\hline \multirow{3}{*}{ Drug abuse } & Multiple partner & $22(44 \%)$ \\
\cline { 2 - 3 } & No & $2(4 \%)$ \\
\cline { 2 - 3 } & Within marriage & $18(36 \%)$ \\
\hline Income & No & $30(60 \%)$ \\
\cline { 2 - 3 } & Yes & $48(96 \%)$ \\
\hline \multirow{2}{*}{ Examination } & Good & $2(4 \%)$ \\
\cline { 2 - 3 } & Low & $16(32 \%)$ \\
\hline \multirow{2}{*}{ Brain MRI } & Normal & $34(68 \%)$ \\
\cline { 2 - 3 } & Abnormal & $32(24 \%)$ \\
\hline \multirow{2}{*}{ History of epilepsy } & Normal & $36(76 \%)$ \\
\cline { 2 - 3 } & Abnormal & $14(28 \%)$ \\
\hline & No & $13(26 \%)$ \\
\cline { 2 - 3 } & Yes & $37(74 \%)$ \\
\hline
\end{tabular}

Table 1: Basic features of the cases. 


\begin{tabular}{|l|l|c|c|}
\hline & & No & $\%$ \\
\hline Cause & Idiopathic & 20 & $40 \%$ \\
& Stroke & 12 & $24 \%$ \\
& CNS infection & 4 & $8 \%$ \\
& Metabolic disorder & 5 & $10 \%$ \\
& Tumor & 4 & $8 \%$ \\
& Trauma & 3 & $6 \%$ \\
& Febrile convulsions & 2 & $4 \%$ \\
\hline Trigger factor & Trauma & 3 & $6 \%$ \\
& Fever & 9 & $18 \%$ \\
& Metabolic disorder & 7 & $14 \%$ \\
& Sleep deprivation & 3 & $6 \%$ \\
& Drug abuse & 2 & $4 \%$ \\
& Ischemia & 1 & $2 \%$ \\
& Poor compliance & 19 & $38 \%$ \\
& Unknown & 6 & $12 \%$ \\
\hline Recurrent SE & No & 44 & $88 \%$ \\
& Yes & 6 & $12 \%$ \\
\hline SE duration & $<60$ min & 28 & $56 \%$ \\
& 60 min-24 hours & 14 & $28 \%$ \\
& $>24$ HOUR & 8 & $16 \%$ \\
\hline AEDs for SE treatment & Combination & 44 & $88 \%$ \\
& Diazepam, phenytoin & 6 & $12 \%$ \\
\hline
\end{tabular}

Table 2: Clinical data of SE.

\begin{tabular}{|c|c|c|c|c|c|c|c|}
\hline & & \multicolumn{2}{|c|}{$\begin{array}{l}\text { No epilepsy history } \\
(\mathrm{n}=13)\end{array}$} & \multicolumn{2}{|c|}{$\begin{array}{l}\text { Positive epilepsy history } \\
(\mathrm{n}=37)\end{array}$} & \multicolumn{2}{|c|}{ Chi-square test } \\
\hline & & No & $\%$ & No & $\%$ & $\mathrm{X}^{2}$ & $\mathrm{P}$ \\
\hline \multirow[t]{2}{*}{ Type of SE } & Convulsive & 11 & $84.6 \%$ & 35 & $94.6 \%$ & \multirow[t]{2}{*}{1.3} & \multirow[t]{2}{*}{.255} \\
\hline & Non convulsive & 2 & $15.4 \%$ & 2 & $5.4 \%$ & & \\
\hline \multirow[t]{6}{*}{ Cause } & Stoke & 5 & $38.5 \%$ & 7 & $18.9 \%$ & \multirow[t]{6}{*}{15} & \multirow{6}{*}{$\begin{array}{l}.011 \\
(\mathrm{~S})\end{array}$} \\
\hline & CNS infection & 2 & $15.4 \%$ & 2 & $5.4 \%$ & & \\
\hline & Metabolic disorder & 3 & $23.1 \%$ & 2 & $5.4 \%$ & & \\
\hline & Trauma & 0 & -- & 3 & $10.3 \%$ & & \\
\hline & Tumor & 1 & $7.7 \%$ & 3 & $10.3 \%$ & & \\
\hline & Idiopathic & 0 & -- & 20 & $54.1 \%$ & & \\
\hline \multirow{8}{*}{$\begin{array}{l}\text { Trigger } \\
\text { factor }\end{array}$} & Fever & 5 & $33.3 \%$ & 4 & $10.8 \%$ & \multirow[t]{8}{*}{19} & \multirow{8}{*}{$\begin{array}{l}.008 \\
(\mathrm{~S})\end{array}$} \\
\hline & Metabolic disorder & 4 & $23.8 \%$ & 3 & $8.1 \%$ & & \\
\hline & Sleep deprivation & 0 & -- & 3 & $8.1 \%$ & & \\
\hline & Drug abuse & 0 & -- & 2 & $5.4 \%$ & & \\
\hline & Ischemia & 1 & $7.7 \%$ & 0 & -- & & \\
\hline & Poor compliance & 0 & -- & 19 & $51.4 \%$ & & \\
\hline & Unknown & 2 & $15.4 \%$ & 4 & $10.8 \%$ & & \\
\hline & Trauma & 1 & $7.7 \%$ & 2 & $5.4 \%$ & & \\
\hline
\end{tabular}

Table 3: Comparison between patients with SE regarding epilepsy history. 


\begin{tabular}{|l|l|c|c|}
\hline$(\mathrm{n}=37)$ & & $\mathrm{N}$ & $\%$ \\
\hline Type of seizure & Generalized & 24 & $64.9 \%$ \\
& Poly morphic & 3 & $8.1 \%$ \\
& Partial & 10 & $27 \%$ \\
\hline AED & Mono & 18 & $48.6 \%$ \\
& Two & 4 & $10.8 \%$ \\
& Poly & 13 & $35.1 \%$ \\
& Untreated & 2 & $5.4 \%$ \\
\hline Drug serum level & Therapeutic & 4 & $10.8 \%$ \\
& Subtherapeutic & 18 & $48.6 \%$ \\
& Can't be assessed & 15 & $40.5 \%$ \\
\hline Etiology & Idiopathic & 20 & $54.1 \%$ \\
& CNS infection & 2 & $5.4 \%$ \\
& Vascular & 9 & $24.3 \%$ \\
& Tumor & 3 & $8.1 \%$ \\
& Trauma & 3 & $8.1 \%$ \\
\hline Seizure frequency before SE & Infrequent & 11 & $37.9 \%$ \\
& Frequent & 18 & $62.1 \%$ \\
\hline
\end{tabular}

Table 4: Distribution of studied group as regard to epilepsy history.

\begin{tabular}{|l|l|l|}
\hline \multirow{2}{*}{ CNS infection } & Encephalitis & Total $(\mathrm{n}=50)$ \\
\cline { 2 - 3 } & Meningitis & $3(6 \%)$ \\
\hline \multirow{3}{*}{ Storke } & Acute & $1(2 \%)$ \\
\cline { 2 - 3 } & Remote & $5(10 \%)$ \\
\hline \multirow{3}{*}{ Mumor } & Primary brain tumor & $7(14 \%)$ \\
\cline { 2 - 3 } & Cerebral metastasis & $2(4 \%)$ \\
\hline \multirow{2}{*}{ Trauma } & Encephalopathy & $2(4 \%)$ \\
\cline { 2 - 3 } & Infantile brain damage & $3(6 \%)$ \\
\cline { 2 - 3 } & Hypertensive encephalopathy & $1(2 \%)$ \\
\hline & Post-traumatic brain damage & $1(2 \%)$ \\
\cline { 2 - 3 } & Intracerebral hemorrhage & $1(2 \%)$ \\
\hline
\end{tabular}

Table 5: Individual etiologies of SE.

\section{DISCUSSION}

Status epilepticus (SE) have a wide clinically and economical influences on the care of cases with critical illness universally as they are frequently accompanying with complicated and prolonged hospitalization and ICU stays. Neurocritical care (NCC) is a quickly rising subject that specializes in the care of cases with critical illness admitted with primary neurological injury. For these cases, the participation of professional NCC clinician has cause significantly better case outcome. Some of the most remarkable NCC subject areas involve seizures and $\mathrm{SE}$, ischemic and hemorrhagic strokes, and traumatic brain injuries (TBI). ${ }^{12}$

SE is a deadly medical emergency which needs fast identification and intervention. The majority of professionals defines SE as 5-min or more of sustained clinical and/or electro-graphic seizure activities or recurrent seizures with no recoveries of consciousness between seizures. The primary aims of SE treatment are to preserve the cases' significant roles, recognize and manage any deadly reasons of $\mathrm{SE}$, and emergently prevent clinically as well as electro-graphic seizure activities. ${ }^{13}$
In this study, we aimed to recognize risk factors and outcomes in SE. We matched in retrospect the prognostic characteristics of SE-episodes with those of NSE in cases managed in a NICU over an interval of 2-yrs.

This was a retrospective cohort study, in which 50 cases had SE-episodes were included, $58 \%$ of the patients were males, $82 \%$ were urban, and $44 \%$ were married, $76 \%$ had abnormal examination, $72 \%$ had normal brain MRI, and $74 \%$ of the patients had history of epilepsy.

Our results are supported by the study of Horváth et al. ${ }^{14}$ which reported that SE diagnosing has been confirmed in 121-cases (males: 61; 50.4\%). As 8cases $(6.6 \%$; males 3 , females: 5 ) had had two or more admissions due to SE, a total of 135 episodes (male: 68, 50.4\%) have been assessed. The cases' ages mean was $64.1 \pm 13.9$-yrs. Built on the age's distribution, $87(71.9 \%)$ of the cases were from 40 to 80 -yrs old. Amid them, the active ages group involved $50(41.3 \%)$ cases.

Another study of Holtkamp et al. ${ }^{7}$ was conducted on a number of 83 episodes in 79 cases (51/83 (61.4\%) females) managed in the NICU satisfied our SE- 
diagnosing criteria. The ages mean was 53.3 (SD 19)-yrs (ranging from 11-94) with one peak in the $4^{\text {th }}$ decade and one more in the 7 th. The vast mainstream of cases involved in this work were adults, with only 2-cases of ages 11 and 16-yrs. Distributions of ages and gender wasn't significantly changed amid the studied groups. SE persevered with admittance to the NICU in $50.6 \%(42 / 83)$ and was ended just previous to admittance in $41 \%(34 / 83)$ of patients. In $8.4 \%$ (7/83) of patients SE happened whereas cases have been managed in the NICU for different causes.

In the current study, we assessed the studied cases as regard clinical characteristics of SE, and found that the commonest cause was idiopathic $(40 \%)$, the commonest trigger factor was poor compliance (38\%). $88 \%$ of the patients were recurrent SE.

In a multicenter cohort by Shorvon and Ferlisi, ${ }^{15}$ reported that SE was most frequently accompanying with cerebro-vascular disorder, substances usage, and CNS inflammations. CNS inflammation was because of infections, auto-immune encephalitis, or cryptogenic. Of these, cryptogenic CNS inflammations causing SE was most challenge to manage and counted a nondependent SRSE risk factor.

Horváth et al. ${ }^{14}$ reported that infections were the main causes of SE in their cohort. Between the cases with preexisting epilepsy, noncompliance, alcoholism and strokes tailed infections by incidence, whereas alcoholism, strokes and cancer were the commonest etiologies between NOSE-cases.

In a Norwegian SE cohort of Ulvin et al. ${ }^{16}$, cerebrovascular disorders, intra-cranial cancers, small levels of AED, and neuro-degenerative disorders have been registered as the commonest reason for SE.

Leppik, ${ }^{17}$ found that strokes to be the reasons of $52.3 \%$ of the patients of SE between the ageing adults and $17.7 \%$ in middle-ages adults.

The SE management, by agreement, happens in phases, which were termed $1^{\text {st }}$ - through $5^{\text {th }}$-line treatment. These phases are really an order of AEDs. $1^{\text {st }}$-line treatment is frequently a benzo. If SE isn't controlled, the sequence lasts with a $2^{\text {nd }} \mathrm{AED}$, that is tailed by a $3^{\text {rd }}, 4^{\text {th }}$, or maybe a $5^{\text {th }}$ AED if required. SE is taken into consideration as RSE when seizure activities lasts afterward receiving therapy with benzos tailed by infusion of a old-style AED. ${ }^{18}$

Furthermore, as regard AEDs for SE treatment, we found that $88 \%$ treated by a combination therapy, while $12 \%$ were treated by Diazepam, phenytoin.

This comes in comparison with the study of Horvath et al. ${ }^{14}$ reported that all recognized cases with epilepsy took 1,2, and 3 or more kinds of AEDS, 34 (52.3\%), 21 (32.3\%), 12 (18.5\%), correspondingly.

Remarkably, if we compared with Horváth et al. ${ }^{19}$ the pattern of AED usage between SE-cases with an epilepsy history with the information of cases with epilepsy in their outpatient care unit, the changes were significant $(\mathrm{p}$-value $=0.0014)(34[52.3 \%]$ versus 894 [69.7\%]; 21 [32.3\%] versus 286 [22.3\%]; 12 [18.5\%] versus 102 [8\%]).

On the other hand, on comparison between patients with SE regarding history of epilepsy, we found that a high significant change was found among the studied groups concerning causes and Trigger factors.

Horváth et al. ${ }^{14}$ reported that a high significant change was found among the study groups in regard to causes and Trigger factors and revealed that between cases with formerly identified epileptic seizures; the commonest reason of SE were infection, strokes, alcoholism and noncompliance whereas between NOSE-cases, infection, alcoholism, strokes and cancer were at the upper of the list. Only $2 / 3$ of the cases with epilepsy were repeatedly managed by an epileptologist previous to SE; these cases all have refractory epilepsy.

In Fernando Gustavo et al. ${ }^{20}$ reported that in formerly epileptic persons, the main reasons of SE are non-compliance to treat or modify AED treatment, accompanying with 20 to $55 \%$ of patients. In their sample, AED non-compliance, removal, or decrease was detected in $19.6 \%$ (43.6\% of formerly epileptic persons), and the rate of death in this group was $11.5 \%(\mathrm{n}=3) .2$ mortalities were connected to cardio-respiratory arrest throughout SE treatments.

Another study of Lui et al. ${ }^{21}$ reported that 29cases(33.3\%) have preceding past of epilepsy whereas 58-cases $(66.7 \%)$ have no preceding epilepsy history earlier the SE episode, cases with no preceding epilepsy history were older in comparison to those with epilepsy history (52 versus 43, $p$ value $=0.009$ ), and a high significant change was found among the groups as regard length of hospital stay, Poor outcome upon discharge, and NCSE.

Furthermore, in the current study, we found that among patients with epilepsy history, $65 \%$ were generalized, $48.6 \%$ were on mono AED and the commonest etiology was idiopathic (54\%).

This comes in comparison with the study of Horváth et al. ${ }^{14}$ which reported that of the 89 -cases with identified epilepsy, $37.1 \%$ have focal seizures with no minor generalizations and $23.6 \%$ have focal seizures with minor generalizations. Between the cases with generalized epilepsy $(39.3 \%)$ seizure sorts were as follow: generalized tonicclonic, myo-clonic and nonattendance, i.e., 33, 1 and 1 patients, correspondingly. Only 1-case have a LennoxGastaut disorder history. Overall, $91 \%$ have epilepsy with symptoms between cases with a past of this disorder. Just below $50 \%$ of patients were focal, and focal epilepsy with minor generalization (4-cases; $30.4 \%$; 2-cases; $19.3 \%$ correspondingly); generalized epilepsy was detected in $34.8 \%$ (47) of cases and mixed-type epilepsies amounts to $15.6 \%$ (2-cases) of SE. 9-cases have been detected with NCSE. Of them, 3 -cases have generalized seizure and 4 cases focal 
epilepsy with minor generalization previous to NCSE.

In a more recent report, Ulvin et al. ${ }^{16}$ revealed that the GCSE-level at $67 \%$ in the nonrefractory SEcases, and at $47 \%$ in RSE.

In the other hand to our findings, the focal form of SE was commonest, just as in the reports by Sutter et al. ${ }^{22}$ and Novy et al. ${ }^{23}$. Probable disagreements between these results can be because of differences in etiology.

On the other hand, Holtkamp et al. ${ }^{7}$ reported that the commonest shape of SE was CPSE (CPSE; 41/83, $49.4 \%)$, tailed by GCSE with fractional onset $(16 / 83$, $19.3 \%)$ and GCSE with no fractional onset $(15 / 83$, $18.1 \%$ ). Simple fractional SE was found in $8.4 \%$ (7/83) of cases.

The current work, being a retrospective investigation, have many limits. Initially, the consequences dependent on the case records. The recording quality and correctness on the descriptions of the seizure on performance differ, contributive to info bias. Then, not all cases presented to the ICU experienced an EEG evaluation in opinion of restricted availability of service. The NCSE diagnosing was restricted to those in which EEG was accomplished, leading to a possible under-estimation of NCSE. As in all retrospective investigation, probable confounders may happen. Cases with SE may have several comorbidities pre-disposing to SE. It was hard to start the causal association of the kind of seizure and the clinical outcomes. In spite of the restrictions, this work was capable to recognize clinical features and predictive issues in cases detected with SE, particularly in persons with de novo SE.

In future, a prospective case control investigation may aid to minimalize bias and confounders in assessing the connotation between the shapes of SE, the etiologies, and the clinical outcomes.

\section{CONCLUSION}

many disorders complicate the picture in daily practice so real-life data are vital so as to understand real cases in the ward. No strict seasonality of SE patients was detected. In our study, the commonest cause of SE was idiopathic, the commonest trigger factor was poor compliance and most of the patients were recurrent SE. This work highlighted the significance of regular care and following-up of cases.

\section{REFERENCES}

1. Laura Mantoan Ritter and Lina Nashef. Newonset refractory status epilepticus (NORSE), Practical Neurology, 10.1136/practneurol-2020002534, 2021; 21(2), 119-27.

2. Kurtz P, Gaspard N, Wahl AS, et al. Continuous electroencephalography in a surgical intensive care unit. Intensive care medicine. 2014;40(2):228-34.

3. Brophy GM, Bell R, Claassen J, et al. Guidelines for the evaluation and management of status epilepticus. Neurocrit Care. 2012;17(1):3-23

4. Trinka E, Cock H, Hesdorffer D, et al. A definition and classification of status epilepticus-Report of the ILAE Task Force on Classification of Status Epilepticus. Epilepsia. 2015;56(10), 1515-23

5. Trinka E, Hofler J and Zerbs A. Causes of status epilepticus. Epilepsia. 2012;53(4):127-38.

6. Shorvon $\mathrm{S}$ and Ferlisi M. The treatment of superrefractory status epilepticus: a critical review of available therapies and a clinical treatment protocol. Brain. 2011:134(10):2802-18.

7. Holtkamp M, Othman J, Buchheim K, et al. Predictors and prognosis of refractory status epilepticus treated in a neurological intensive care unit. $J$ Neurol Neurosurg Psychiatry. 2005;76(4):534-9.

8. Sculier, C., Gaínza-Lein, M., Sánchez Fernández, I., et al (2018). Long-term outcomes of status epilepticus: A critical assessment. Epilepsia. 2018;2(2):155-169.

9. Hocker S, Tatum WO, LaRoche S, et al. Refractory and super-refractory status epilepticus--an update. Curr Neurol Neurosci Rep. 2014 14(6):452.

10. Singh, S. P., Agarwal, S and Faulkner, M. (2014). Refractory status epilepticus. Annals of Indian Academy of Neurology. 2014;17(1), 32-6.

11. Power KN, Gramstad A, Gilhus NE, Engelsen BA. Prognostic factors of status epilepticus in adults. Epileptic Disorders. 2016;18(3):297-304.

12. McNett M., Moran C and Johnson H. EvidenceBased Review of Clinical Trials in Neurocritical Care. AACN Adv. Crit. Care. 2018;29:195-203.

13. Riviello, J.J., Claassen, J., LaRoche, S.M. et al. Treatment of Status Epilepticus: An International Survey of Experts. Neurocrit Care. 2013;18;193200.

14. Horváth L, Fekete I, Molnár M, et al. (2019) The Outcome of Status Epilepticus and Long-Term Follow-Up. Front. Neurol. 2019;10:427.

15. Shorvon S and Ferlisi M. The treatment of superrefractory status epilepticus: A critical review of available therapies and a clinical treatment protocol. Brain J. Neurol. 2011;134:2802-18.

16. Ulvin LB, Heuser K, Olsen KB, et al. Factors associated with refractoriness and outcome in an adult status epilepticus cohort. Seizure. 2018; 61:111-8

17. Leppik IE. Status epilepticus in the elderly. Epilepsia. 2018;59(2):140-3.

18. Brophy GB, Bell R, Alldredge A, et al. Neurocritical Care Society Status Epilepticus Guideline Writing Committee. Guidelines for the evaluation and management of status epilepticus. Neurocrit Care. 2012;17:3-23.

19. Horváth L, Fekete K, Márton S and Fekete I. Outcome of antiepileptic drug treatment of 1282 patients with epilepsy, their pharmacovigilance reports and concomitant medication on CNS in an East-Hungarian adult database. J Neurol Sci. 2016; 369:220-6. 
20. Fernando Gustavo S, Bustamante, Guilherme de Oliveira, et al. (2015). Short-term mortality and prognostic factors related to status epilepticus. Arquivos de Neuro-Psiquiatria. 2015; 73(8), 6705.

21. Lui H, Kwok Fai, Huia Wing, et al. De novo status epilepticus is associated with adverse outcome: An 11-year retrospective study in Hong Kong, Seizure. 2016; 1:40:42-5.

22. Sutter R, Kaplan PW, Marsch S, et al. Early predictors of refractory status epilepticus: an international two-center study. Eur J Neurol. 2015;22:79-85.

23. Novy J, Logroscino $G$ and Rossetti AO. Refractory status epilepticus: a prospective observational study. Epilepsia. 2010; 51:251-6. 